\title{
The Size-Ramsey Number of 3-uniform Tight Paths
}

\author{
Jie Han* Yoshiharu Kohayakawa ${ }^{\dagger} \quad$ Shoham Letzter ${ }^{\ddagger}$ \\ Guilherme Oliveira Mota ${ }^{\S} \quad$ Olaf Parczyk ${ }^{\mathrm{I}}$
}

Received 23 June 2020; Published 11 June 2021

\begin{abstract}
Given a hypergraph $H$, the size-Ramsey number $\hat{r}_{2}(H)$ is the smallest integer $m$ such that there exists a hypergraph $G$ with $m$ edges with the property that in any colouring of the edges of $G$ with two colours there is a monochromatic copy of $H$. We prove that the size-Ramsey number of the 3-uniform tight path on $n$ vertices $P_{n}^{(3)}$ is linear in $n$, i.e., $\hat{r}_{2}\left(P_{n}^{(3)}\right)=O(n)$. This answers a question by Dudek, La Fleur, Mubayi, and Rödl for 3uniform hypergraphs [On the size-Ramsey number of hypergraphs, J. Graph Theory $\mathbf{8 6}$ (2016), 417-434], who proved $\hat{r}_{2}\left(P_{n}^{(3)}\right)=O\left(n^{3 / 2} \log ^{3 / 2} n\right)$.
\end{abstract}

Key words and phrases: size-Ramsey number, hypergraph, tight path

\section{Introduction}

For hypergraphs $G$ and $H$ and an integer $s$, we denote by $G \rightarrow(H)_{s}$ the property that in any $s$-colouring of the edges of $G$ there is a monochromatic copy of $H$. The $s$-colour size-Ramsey number $\hat{r}_{s}(H)$ is

$$
\hat{r}_{s}(H):=\min \left\{|E(G)|: G \rightarrow(H)_{s}\right\} .
$$

*Partially supported by the Simons Collaboration Grant for Mathematicians \#630884.

†Partially supported by CNPq (311412/2018-1, 423833/2018-9) and FAPESP (2018/04876-1, 2019/13364-7)

$\ddagger$ Research supported by the Royal Society.

${ }^{\S}$ Partially supported by CNPq (304733/2017-2, 428385/2018-4) and FAPESP (2018/04876-1, 2019/13364-7).

IPartially supported by Technische Universität Ilmenau, the Carl Zeiss Foundation, and the DFG (Grant PA 3513/1-1). The cooperation of the authors was supported by a joint CAPES/DAAD PROBRAL project (Proj. 430/15, 57350402, 57391197). This study was financed in part by CAPES, Coordenação de Aperfeiçoamento de Pessoal de Nível Superior, Brazil, Finance Code 001. FAPESP is the São Paulo Research Foundation. CNPq is the National Council for Scientific and Technological Development of Brazil. 


\section{J. Han, Y. Kohayakawa, S. Letzter, G. O. Mota and O. Parczy}

For the $n$-vertex path $P_{n}$, Erdős [11] asked if $\hat{r}_{2}\left(P_{n}\right)=O(n)$, which was answered positively by Beck [3] using the probabilistic method. An explicit construction for the same results was given by Alon and Chung [1]. Many successive improvements led to the currently best known bounds $3.75 n-o(n) \leq \hat{r}_{2}\left(P_{n}\right) \leq 74 n$ (see, e.g., [3, 6, 10, 2] for lower bounds, and [3, 9, 14, 10] for upper bounds). For $s \geq 2$ colours, Dudek and Prałat [10] and Krivelevich [13] proved that there are constants $c$ and $C$ such that $c s^{2} n \leq \hat{r}_{s}\left(P_{n}\right) \leq C s^{2}(\log s) n$.

The systematic investigation of size-Ramsey questions for hypergraphs was initiated by Dudek, La Fleur, Mubayi, and Rödl [8]. Besides cliques and trees, they studied generalisations of paths.

We say that an $r$-uniform hypergraph is an $\ell$-path if there exists an ordering of its vertices such that every edge is composed of $r$ consecutive vertices, two (vertex-wise) consecutive edges share exactly $\ell$ vertices, and every vertex is contained in an edge. For $1 \leq \ell \leq r-1$, let $P_{n, \ell}^{(r)}$ denote the $r$-uniform $\ell$-path on $n$ vertices and for the tight path, where $\ell=r-1$, we write $P_{n}^{(r)}$. Dudek, La Fleur, Mubayi, and Rödl [8] deduced from Beck's result [3] that $\hat{r}_{2}\left(P_{n, \ell}^{(r)}\right)=O(n)$, when $1 \leq \ell \leq r / 2$. Furthermore, they proved that $\hat{r}_{2}\left(P_{n}^{(r)}\right)=O_{r}\left(n^{r-1-\alpha} \log ^{1+\alpha} n\right)$ with $\alpha=(r-2) /\left(\left(\begin{array}{c}r-1 \\ 2\end{array}\right)+1\right)$, which gives $\hat{r}_{2}\left(P_{n}^{(3)}\right)=O\left(n^{3 / 2} \log ^{3 / 2} n\right)$.

This was improved and extended to more colours by Lu and Wang [15], who showed that $\hat{r}_{s}\left(P_{n}^{(r)}\right)=$ $O_{r}\left(s^{r}(n \log n)^{r / 2}\right)$ for $s \geq 2$ colours. Dudek, La Fleur, Mubayi, and Rödl [8] asked if $\hat{r}_{2}\left(P_{n}^{(r)}\right)=O_{r}(n)$ for $r \geq 3$. We answer this question for 3-uniform hypergraphs by proving the following result.

Theorem 1. The 2-colour size-Ramsey number of the 3-uniform tight path is

$$
\hat{r}_{2}\left(P_{n}^{(3)}\right)=O(n)
$$

Trivially, we need at least $n$ edges, so this is asymptotically optimal. As observed in [8], bounds on size-Ramsey numbers for some uniformity can be used to obtain bounds for larger uniformities. We obtain the following corollary.

Corollary 2. For any integer $r$ such that $3 \mid r$, the 2-colour size-Ramsey number of the $r$-uniform $(2 r / 3)$-path is

$$
\hat{r}_{2}\left(P_{n, 2 r / 3}^{(r)}\right)=O(n)
$$

To see this, take the graph given by Theorem 1 and replace every vertex by a set of $r / 3$ vertices. Then each 3-edge naturally gives an $r$-edge, and every 3 -uniform tight path becomes an $r$-uniform $(2 r / 3)$-path.

Our proof combines new ideas and the method developed by Clemens, Jenssen, Kohayakawa, Morrison, Mota, Reding, and Roberts [7] for estimating the size-Ramsey number of powers of paths (see also $[5,12])$. It is plausible that ideas from $[12,5]$ may provide a strategy to solve the case with $s \geq 3$ colours. However, the question whether the size-Ramsey number of a tight path is linear for hypergraphs with uniformity $r \geq 4$ remains open and requires additional ideas.

\section{Preliminaries}

In this short section, we give a sketch of our proof of Theorem 1 and state two simple lemmas about random graphs. 


\subsection{Sketch of the proof of Theorem 1}

We will first sketch a proof for $\hat{r}_{2}\left(P_{n}\right)=O(n)$. It is not hard (cf. Lemmas 3 and 4 below) to obtain a graph $G$ with $O(n)$ edges such that for any two sufficiently large and disjoint sets of vertices $A$ and $B$ there is a path of length $n$ alternating between $A$ and $B$. Given such a graph $G$, we show that $G \rightarrow\left(P_{n}\right)_{2}$. Consider an arbitrary 2-colouring of the edges of $G$ with colours blue and red. If there is no blue $P_{n}$ in $G$ we can show (cf. Lemma 6 below) that there are two sets $A$ and $B$ of size at least $n$ with no blue edges in between. By the property of $G$ mentioned above there exists a $P_{n}$ alternating between $A$ and $B$, which unequivocally has to be red.

For the proof of Theorem 1 we follow, in principle, the same strategy. Based on a blow-up of a power of a similar graph $G$, we define a 3-uniform hypergraph $H$ and claim that $H \rightarrow\left(P_{n}^{(3)}\right)_{2}$. We define an auxiliary (generalised) graph $F$ on $V(G)$, which has 2- and 3-edges, such that a long path in $F$ gives a blue $P_{n}^{(3)}$ in $H$. If $F$ does not contain a long path, then we find a family of disjoint sets such that no edge of $F$ lies between these sets (cf. Lemma 6). Then by the properties of $G$ there exists a path in $G$ alternating through these sets. As there are no edges of $F$ 'interfering' with this path, we are able to turn it into a red $P_{n}^{(3)}$ in $H$.

In the next section we provide the lemmas needed to obtain $G$. Afterwards, in Section 3 we introduce the notion of (2,3)-graphs, which, as can be seen above, plays a key role in our argument. Finally, we prove Theorem 1 in Section 4.

\subsection{Sparse graphs with many long paths}

The following two lemmas are proved in [7]. Basically, together they imply that for every $k$ and $n$ there exists a graph $G$ with $O_{k}(n)$ edges such that, for any disjoint sets of vertices $A_{1}, \ldots, A_{k+1}$ that are large enough, there exists a path of length $n$ 'alternating' through these sets.

Lemma 3 ([7, Lemma 3.1]). For every pair of positive constants $\varepsilon$ and a, there is a constant $b$ such that, for any large enough $n$, there is a graph $H$ with $v(H)=$ an and $\Delta(H) \leq b$ such that the following holds:

$\left(\mathrm{P} 1_{n}\right)$ For every pair of disjoint sets $S, T \subseteq V(H)$ with $|S|,|T| \geq \varepsilon$, we get $e_{H}(S, T)>0$.

Lemma 4 ([7, Lemma 3.5]). For every integer $k \geq 1$ and every $\varepsilon>0$ there exists an integer a such that the following holds. Let $H$ be a graph on at least am vertices such that for every pair of disjoint sets $S$, $T \subseteq V(H)$ with $|S|,|T| \geq \varepsilon m$ we have $e_{H}(S, T)>0$. Then the following holds:

$\left(\mathrm{P} 2_{m}\right)$ For every family $A_{1}, \ldots, A_{k+1} \subseteq V(H)$ of pairwise disjoint sets each of size at least $\varepsilon a m$, there is a path $P_{m}=\left(x_{1}, \ldots, x_{m}\right)$ in $H$ with $x_{i} \in A_{j}$ for all $1 \leq i \leq m$, where $j \equiv i(\bmod k+1)$.

Note that the hypothesis on $H$ in Lemma 4 is $\left(\mathrm{P} 1_{m}\right)$ from Lemma 3. Therefore, roughly speaking, Lemma 4 tells us that $\left(\mathrm{P} 1_{m}\right)$ implies $\left(\mathrm{P} 2_{m}\right)$.

\section{3 (2,3)-graphs}

In this section we introduce a structure that helps us to transfer some ideas from the graph case to the hypergraphs setting. A $(2,3)$-graph $F=(V, E)$ consists of a set of vertices $V$ and a set $E$ of 2-edges of 


\section{J. Han, Y. Kohayakawa, S. Letzter, G. O. Mota And O. Parczy}

the form $\{u, v\}$ and 3-edges of the form $(\{u, v\}, w)$, for distinct vertices $u, v, w \in V$. For simplicity we will write $u v$ for $\{u, v\}$ and $u v(w)$ for $(\{u, v\}, w)$. A sequence of vertices $P=\left(x_{1}, \ldots, x_{m}\right)$ is a (2,3)-path of length $m$ in $F$ if for every $i=1, \ldots, m-1$ either $x_{i} x_{i+1} \in E$ or $x_{i} x_{i+1}\left(w_{i}\right) \in E$ for some $w_{i} \in V \backslash\left\{x_{1}, \ldots, x_{m}\right\}$, with all the $w_{i}$ distinct.

Given pairwise disjoint sets $V_{1}, \ldots, V_{k+1}$, we say that an edge $u v \in E(F)(u v(w) \in E(F))$ is a transversal with respect to $V_{1}, \ldots, V_{k+1}$, if $u$ and $v(u, v$, and $w)$ are in different sets $V_{i}$. When the sets $V_{1}, \ldots, V_{k+1}$ are clear from the context we say that the edge is a transversal.

We want to prove that if a sufficiently large $(2,3)$-graph $F=(V, E)$ contains no $(2,3)$-path with $n$ vertices, then there exist large disjoint sets $V_{1}, \ldots, V_{k} \subseteq V$ such that $E$ contains no transversals and that there is no edge $u v(w)$ with $u \in V_{1} \cup \cdots \cup V_{k-1}$ and $v, w \in V_{k}$. The last property is only required to support our inductive proof. To prove this we use a Depth First Search (DFS) algorithm. For example, Ben-Eliezer, Krivelevich, and Sudakov [4] used a DFS algorithm to find long paths in expanding graphs to obtain bounds on the size-Ramsey number of directed paths. Their algorithm traverses the vertices of the input graph and maintains a set $S$ of vertices that are fully dealt with, a set $U$ of currently active vertices, and a set $J$ of vertices that were not considered so far. The set $U$ always spans a path and in every step, if at all possible, this path is extended by adding a vertex from $J$. Otherwise, the last vertex of the path is removed and added to $S$. It is immediate that there cannot be any edges between $S$ and $J$ and if $U$ stays small, then at some point during the execution both $S$ and $J$ are large.

We adapt this algorithm to the setting of $(2,3)$-graphs (see Algorithm 1 below). As in the graph case we greedily extend a (2,3)-path (preferring 2-edges over 3-edges) and backtrack if it gets stuck. We will now give the details of our algorithm. The input is a $(2,3)$-graph $F=(V, E)$, disjoint subsets of vertices $V_{1}, \ldots, V_{k}$, and an ordering of the vertices $V=\left\{v_{1}, \ldots, v_{N}\right\}$. During the algorithm we maintain sets $S, T$, $W_{S}, W_{U}, T_{i}$ for $i \in[k]$ and a $(2,3)$-path $U$ as follows:

- $S \subseteq V^{\prime}$ is the set of vertices that are fully dealt with.

- $W_{S} \subseteq V$ is the set of vertices $w$ that were 'used' by vertices from $S$.

- $U$ contains the currently active vertices in a $(2,3)$-path.

- $W_{U} \subseteq V$ is the set of vertices $w$ that are 'used' by the path $U$.

- $T_{1} \cup \cdots \cup T_{k}$ are disjoint and $T_{i} \subset V_{i}$ for $i \in[k]$.

In every step of the algorithm, either the (2,3)-path $U$ is extended by adding a vertex from $T_{k}$ to it or this is not possible, and the last vertex from $U$ is removed and put into $S$. While the algorithm runs, after each execution of the while loop, we have the following invariants, where $m$ is the length of the (2,3)-path $U$ :

(A1) $U=\left(u_{1}, \ldots, u_{m}\right)$ is a $(2,3)$-path and $W_{U}$ is the set of the vertices $w$ in the edges $u_{i} u_{i+1}(w)(1 \leq i<m)$ in the (2,3)-path $U$.

(A2) $S, U \subseteq V_{k}, W_{U} \subseteq T_{1} \cup \cdots \cup T_{k-1}, T_{i} \subseteq V_{i}$ for $i \in[k],\left|W_{S}\right| \leq|S|$, and $\left|W_{U}\right| \leq \max \{0, m-1\}$.

This process is described in Algorithm 1.

Lemma 5. Algorithm 1 terminates and Properties (A1) and (A2) hold throughout. 


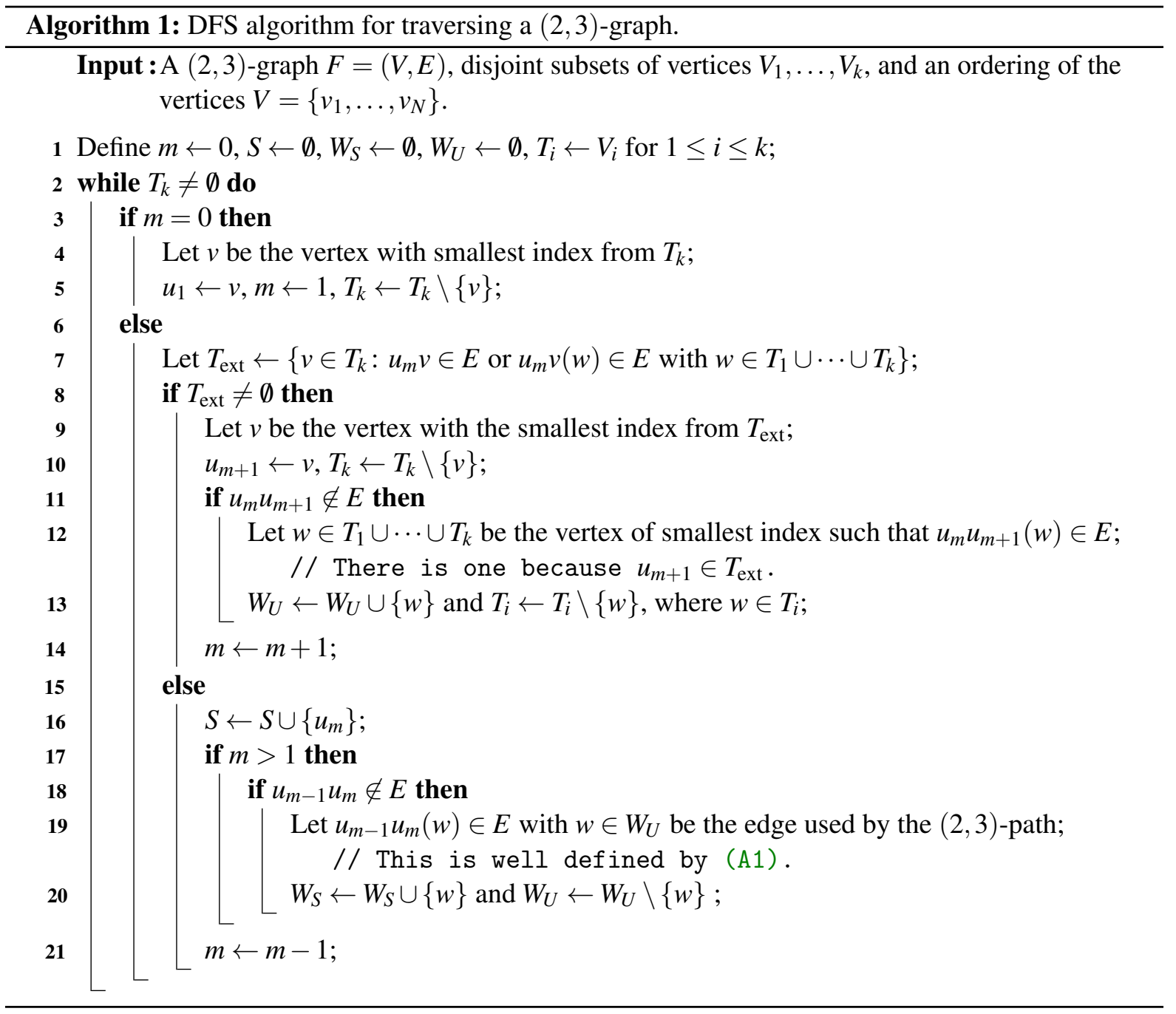




\section{J. Han, Y. Kohayakawa, S. Letzter, G. O. Mota And O. Parczy}

Proof of Lemma 5. Observe that (A1) and (A2) hold when we initialise the sets and put $m=0$ on line 1. Assume that we are in some step of the algorithm, where (A1) and (A2) hold and we have vertices $U=\left(u_{1}, \ldots, u_{m}\right)$ forming a $(2,3)$-path (this is true because of (A1)).

Now we consider the next execution of the while loop. We know from (A1) that $W_{U}$ contains exactly the vertices used in the edges $u_{i} u_{i+1}(w)$ for $i=1, \ldots, m-1$. Either we extend the path by an edge $u_{m} v$ or $u_{m} v(w)$ (lines 7 and 10) where $w$ is added to $W_{U}$ if needed (line 13), or we remove an edge $u_{m-1} u_{m}$ or $u_{m-1} u_{m}(w)$ (lines 16 and 20) where $w$ is removed from $W_{U}$ if needed (line 20). Therefore, (A1) still holds.

For (A2) it is easy to see that $T_{i} \subseteq V_{i}$ for $i \in[k]$, as in the beginning of the execution we have $T_{i}=V_{i}$ and no vertex is added to $T_{i}$. Also, since every $w$ in $W_{U}$ comes from $T_{1} \cup \cdots \cup T_{k}$, we have $W_{U} \subseteq T_{1} \cup \cdots \cup T_{k-1}$ (see lines 7 and 20). Since every vertex of $S$ comes from $U$ (line 16) and every vertex of $U$ comes from $T_{k} \subset V_{k}$ (lines 5, 7 and 10), which implies that $S, U \subset V_{k}$. To prove that $\left|W_{S}\right| \leq|S|$, it is enough to observe that line 20 can only be executed after an execution of line 16. Similarly, we have $\left|W_{U}\right| \leq m-1$ with $m \geq 2$, because line 13 can only be executed after an execution of line 10 , and $\left|W_{U}\right|=0$ with $m=1$, because on line 5 nothing is added to $W_{U}$. Thus, (A2) also remains true.

It remains to show that the algorithm terminates. In every execution of the while loop, either one vertex from $T_{k} \subseteq V_{k}$ is added to the path $U$ (lines 5 and 10) or moved from $U$ to $S$ (line 16). Therefore, after at most $2\left|V_{k}\right|$ steps we have $T=\emptyset$, and the algorithm terminates.

We are ready to prove the aforementioned result on (2,3)-graphs $F$ with no long (2,3)-paths.

Lemma 6. Let $k, c$ and $n$ be positive integers and let $F=(V, E)$ be a $(2,3)$-graph on at least $5^{k-1}$ cn vertices. If $F$ contains no $(2,3)$-path with $n$ vertices, then there exist disjoint sets $V_{1}, \ldots, V_{k} \subseteq V$ of size at least cn such that no edge from $E$ is a transversal and there is no edge $u v(w)$ with $u \in V_{1} \cup \cdots \cup V_{k-1}$ and $v, w \in V_{k}$.

Proof. We prove the result by induction on $k$. For $k=1$ the result follows by putting $V_{1}=V(F)$. Thus let $k \geq 1$ and assume the statement holds for $k$.

To prove the result for $k+1$, let $F$ be a $(2,3)$-graph on $5^{k} c n$ vertices which does not have a path of length $n$. In particular, $F$ does not have a path of length $5 n$, and by the assumption on $k$, there exist disjoint sets $V_{1}, \cdots, V_{k}$, each of size $5 c n$, such that no edge is a transversal, and there is no edge $u v(w)$ with $u \in V_{1} \cup \cdots \cup V_{k-1}$ and $v, w \in V_{k}$. We run Algorithm 1 with input $F, k$ and $V_{1}, \ldots, V_{k}$.

First, we prove that at any point in the execution of the algorithm, no edge is a transversal with respect to $T_{1} \cup \cdots \cup T_{k}, S$. Suppose for a contradiction that at some point there is an edge which is a transversal. Note that $T_{k} \subseteq V_{k}$ and $S \subseteq V_{k}$. If $u v$ is this edge, then by the induction hypothesis and without loss of generality we have $u \in S$ and $v \in T_{k}$. This implies that when $u$ was moved from $U$ to $S$ (line 16), the set $U$ could have been extended, which means that $T_{\text {ext }} \neq \emptyset$ and line 16 would not have been executed, a contradiction. Now, assume $u v(w)$ is the transversal. Since $T_{i} \subseteq V_{i}$ for $i \in[k]$ and $S \subseteq V_{k}$, we have $w \in T_{1} \cup \cdots \cup T_{k-1}$. Again, by the induction hypothesis and without loss of generality we have $u \in S$ and $v \in T_{k}$. Similarly as when we have an edge $u v$, at the time $u$ was moved from $U$ to $S$, the set $U$ could have been extended, a contradiction.

Now we prove that at any point in the execution of the algorithm, there is no edge $u v(w)$ with $u \in T_{1}, \ldots, T_{k-1}, S$ and $v, w \in T_{k}$. Suppose for a contradiction that at some point there is such edge $u v(w)$. 
By the induction hypothesis we have $u \in S$ and $v, w \in T_{k}$, which again gives a contradiction as $U$ could have been extended.

Note that since $F$ has at least $5^{k-1} c n$ vertices and no (2,3)-path with $n$ vertices, we have $|S|=c n$ at some point of the execution of Algorithm 1. Let $U, W_{U}, S, W_{S}$ and $T_{1}, \ldots, T_{k}$ be the sets at that moment. Note that $\left|W_{S}\right| \leq|S|=c n$ and, since there is no $n$-vertex (2,3)-path in $F$, we have $|U|,\left|W_{U}\right| \leq n$. Therefore, $\left|T_{i}\right| \geq\left|V_{i}\right|-2 c n \geq c n$ for $i \in[k-1]$ and $\left|T_{k}\right| \geq\left|V_{k}\right|-4 c n \geq c n$. Put $V_{i}^{\prime}=T_{i}$ for $i \in[k-1]$, $V_{k}^{\prime}=S$ and $V_{k+1}^{\prime}=T_{k}$. The sets $V_{1}^{\prime}, \ldots, V_{k+1}^{\prime}$ satisfies the requirements of the lemma.

\section{Proof of Theorem 1}

In this section we prove our main theorem. We first define the following constants:

$$
\ell:=17, \quad k:=2 \ell, \quad \varepsilon:=1 /(k+1), \quad t:=8 k+40 k^{2}+5, \quad \text { and } \quad t^{\prime}:=r_{2}\left(K_{t}^{(3)}\right),
$$

where $r_{s}\left(K_{t}^{(3)}\right):=\min \left\{n: K_{n}^{(3)} \rightarrow\left(K_{t}^{(3)}\right)_{s}\right\}$ is the classical Ramsey-number for hypergraphs. We start by obtaining a graph $G$ with bounded maximum degree and some nice pseudorandom properties. Let $a_{\mathrm{L} .4}$ be large enough to apply Lemma 4 with $k$ and $\varepsilon$ and set

$$
c:=\varepsilon a_{\mathrm{L} .4} \ell \text {. }
$$

Let $a_{\mathrm{L} .6}=5^{k}$ and note that $a_{\mathrm{L} .6}$ is large enough to apply Lemma 6 with $k+1, c$ and $n$ and set

$$
a:=2 a_{\mathrm{L} .6} .
$$

Lemma 3 applied with $\varepsilon$ and $a$ provides a constant $b$. Let $n$ be sufficiently large. Then, from Lemma 3 we know that there is a graph $G$ on $a n$ vertices with maximum degree $b$ such that $\left(\mathrm{P} 1_{n}\right)$ holds. Fix such a graph $G$.

Now let $G^{k}\left(t^{\prime}\right)$ be the graph obtained from $G^{k}$ - the $k$-th power of $G$ - by replacing every vertex by a $K_{t^{\prime}}$ and every edge by a $K_{t^{\prime}, t^{\prime}}$. Finally, $H$ is the 3-uniform hypergraph with vertex set $V\left(G^{k}\left(t^{\prime}\right)\right)$ and a triple of vertices $x y z$ is an edge in $H$ if and only if $x y z$ forms a triangle in $G^{k}\left(t^{\prime}\right)$. For every $v \in V(G)$ we denote by $H(v)$ the corresponding cluster consisting of a $K_{t^{\prime}}^{(3)}$ in $H$. We claim that $H \rightarrow\left(P_{n}^{(3)}\right)_{2}$. Since $|V(H)|=a t^{\prime} n$ and $\Delta(H) \leq b^{2 k+2} t^{\prime 3}$, this would prove Theorem 1 .

The rest of the proof is devoted to proving that $H \rightarrow\left(P_{n}^{(3)}\right)_{2}$. Fix a 2-colouring of the triples of $H$. As $t^{\prime} \geq r_{2}\left(K_{t}^{(3)}\right)$ for every $v \in V(G)$ the cluster $H(v)$ either contains a red or blue copy of $K_{t}^{(3)}$. W.l.o.g. there is a set of vertices $V \subseteq V(G)$ with $|V| \geq a n / 2=a_{\mathrm{L} .6} n$ such that for all $v \in V$ the cluster $H(v)$ contains a blue copy of $K_{t}^{(3)}$, which we denote by $H^{\prime}(v)$. We let $H^{\prime} \subseteq H$ be the 3-graph induced by the clusters $H^{\prime}(v)$ for $v \in V$.

We will define an auxiliary $(2,3)$-graph $F$ on the vertex set $V$, whose edges will indicate that we can walk between the clusters using blue triples of $H^{\prime}$. Formally, for $u, v \in V$ a $(2,2)$-connector between the clusters $H^{\prime}(u)$ and $H^{\prime}(v)$ consists of four vertices $x_{1}, x_{2} \in H^{\prime}(u)$ and $y_{1}, y_{2} \in H^{\prime}(v)$ such that $x_{1} x_{2} y_{1}$ and $y_{1} y_{2} x_{1}$ are triples of $H$. Similarly, for $u, v, w \in V$ a $(2,1,2)$-connector between the clusters $H^{\prime}(u)$ and $H^{\prime}(v)$ through $H^{\prime}(w)$ consists of five vertices $x_{1}, x_{2} \in H^{\prime}(u), z \in H^{\prime}(w)$, and $y_{1}, y_{2} \in H^{\prime}(v)$ such that $x_{1} x_{2} z, x_{1} z y_{1}$, and $z y_{1} y_{2}$ are triples of $H$; see Figure 1 . We then define a $(6,6)$-connector $((6,3,6)$-connector $)$ between 


\section{J. Han, Y. Kohayakawa, S. Letzter, G. O. Mota And O. Parczy}
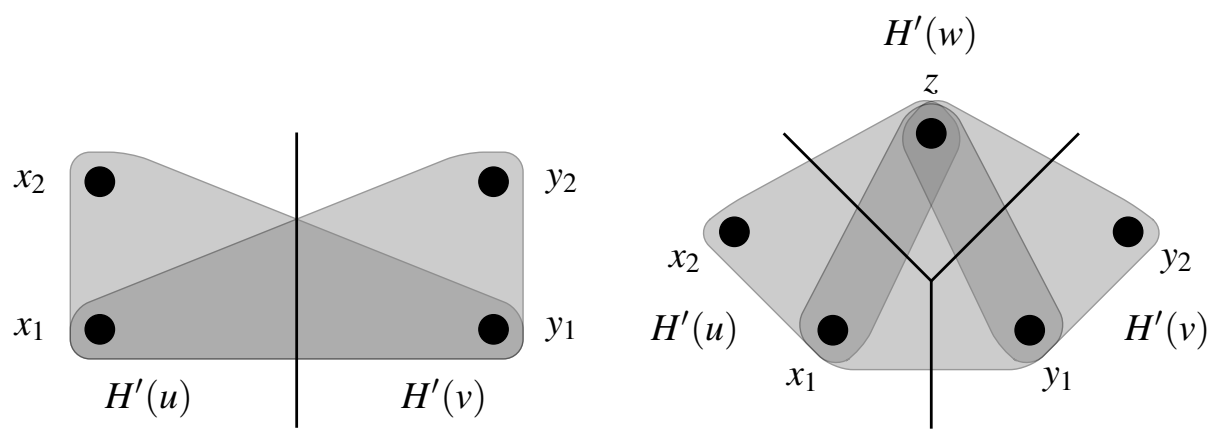

Figure 1: A (2,2)-connector and a $(2,1,2)$-connector.

$H^{\prime}(u)$ and $H^{\prime}(v)$ (through $\left.H^{\prime}(w)\right)$ as the disjoint union of three $(2,2)$-connectors $((2,1,2)$-connectors) between $H^{\prime}(u)$ and $H^{\prime}(v)$ (through $H^{\prime}(w)$ ).

Let $F$ be a (2,3)-graph on the vertex set $V$ with the following two types of edges:

(i) $u v \in E(F)$ if and only if there is a $(6,6)$-connector in blue between the corresponding clusters $H^{\prime}(u)$ and $H^{\prime}(v)$;

(ii) $u v(w) \in E(F)$ if and only if there is a $(6,3,6)$-connector in blue between the corresponding clusters $H^{\prime}(u)$ and $H^{\prime}(v)$ through $H^{\prime}(w)$.

Suppose that $F$ contains a (2,3)-path on $n$ vertices $v_{1}, \ldots, v_{n}$ with the $w_{i}$ vertices all distinct for the 3-edges. We can turn this (2,3)-path into a blue tight path $P_{n}^{(3)}$ in $H$ as follows. First, by following the (2,3)-path for $i=1, \ldots, n-1$, we choose a $(2,2)$-connector between $H^{\prime}\left(v_{i}\right)$ and $H^{\prime}\left(v_{i+1}\right)$ if $v_{i} v_{i+1} \in E(F)$ or a $(2,1,2)$-connector between $H^{\prime}\left(v_{i}\right)$ and $H^{\prime}\left(v_{i+1}\right)$ through $H^{\prime}\left(w_{i}\right)$ if $v_{i} v_{i+1}\left(w_{i}\right) \in E(F)$ in such a way that they are all pairwise vertex-disjoint. This is possible, because we have $(6,6)$-connectors and $(6,3,6)$ connectors available and two vertices $y_{1}$ and $y_{2}$ from the previous connector can occupy at most two out of the three disjoint copies of connectors that are provided. Then, for each of the clusters with $y_{1}, y_{2}$ and $x_{1}, x_{2}$ the vertices of the connectors within that cluster, we use the edges $y_{1} y_{2} x_{2}$ and $y_{2} x_{2} x_{1}$ to connect both connectors. As the connectors only use blue edges and all edges within the clusters are blue this is a tight path only using blue edges. Thus, in this case, we are able to obtain a blue $P_{n}^{(3)}$ in $H$ and we are done.

We assume that $F$ contains no (2,3)-path on $n$ vertices. From Lemma 6, there exist pairwise disjoint sets $V_{1}, \ldots, V_{k+1} \subseteq V$ of size at least $c n$ such that no edge from $E(F)$ is a transversal. We may assume that all these sets $V_{j}$ have size exactly $c n$. Let $G^{\prime}=G\left[V_{1} \cup \cdots \cup V_{k+1}\right]$ and set $m:=\ell n$.

We now want to find a path $P_{m}$ alternating through $V_{1}, \ldots, V_{k+1}$ with edges in $G^{\prime} \subseteq G$ using Lemma 4 . Since $c=\varepsilon a_{\mathrm{L} .4} \ell$ and $\varepsilon=1 /(k+1)$, we have $\left|V\left(G^{\prime}\right)\right|=(k+1) c n=a_{\mathrm{L} .4} l n=a_{\mathrm{L} .4} m$. Also, we have $\left|V_{i}\right|=c n=\varepsilon a_{\mathrm{L} .4} m$ for $1 \leq i \leq k+1$. As $G^{\prime}$ is an induced subgraph of $G$ and property $\left(\mathrm{P} 1_{n}\right)$ holds in $G$, property $\left(\mathrm{P} 1_{m}\right)$ does hold for $G^{\prime}$. Therefore, by Lemma 4, we conclude that there is a path $P_{m}=P_{\ell n}$ with vertices alternating through $V_{1}, \ldots, V_{k+1}$ and with edges in $G^{\prime} \subseteq G$. 
This path $P_{\ell n}$ gives us the $k$ th power $P_{\ell n}^{k}$ in $G^{k}$. By the choice of $V_{1}, \ldots, V_{k+1}$ no edge of $P_{\ell n}^{k}$ is from $E(F)$ and also no triangle in $P_{\ell n}^{k}$ induces an edge $u v(w) \in E(F)$. It remains to turn this $P_{\ell n}^{k}$ into a red $P_{n}^{(3)}$ in $H^{\prime}$.

Claim 7. If there is a $P_{\ell n}^{k}$ in $G^{k}$ that does not contain any edges from $F$, then there is a red $P_{n}^{(3)}$ in $H^{\prime}$.

Let $P_{\ell n}^{k}=\left(v_{1}, \ldots, v_{\ell n}\right)$ and recall $H^{\prime}\left(v_{i}\right)$ is the cluster in $H^{\prime}$ corresponding to the vertex $v_{i}$ for $i=$ $1, \ldots, \ell n$. We want to remove all vertices of $H^{\prime}$ which belong to blue $(2,2)$-connectors and $(2,1,2)$ connectors from clusters along edges and triangles of $P_{\ell n}^{k}$. In $P_{\ell n}^{k}$ every vertex $v_{i}$ is incident to at most $2 k$ other vertices in $\left\{v_{1}, \ldots, v_{\ell n}\right\}$ ( $2 k$ is the maximum degree of the $v_{i}$ in $P_{\ell n}^{k}$ ). Also, every $v_{i}$ is contained in at most $4 k^{2}$ triangles of $P_{\ell n}^{k}$ together with two other vertices in $\left\{v_{1}, \ldots, v_{\ell n}\right\}$.

Let $u$ and $v$ be neighbours in $P_{\ell n}^{k}$. Since there is no blue $(6,6)$-connector between $H^{\prime}(u)$ and $H^{\prime}(v)$, there are at most two $(2,2)$-connectors that do not overlap between $H^{\prime}(u)$ and $H^{\prime}(v)$, which can both be deleted by removing at most 4 vertices in each cluster. Let $u, v$, and $w$ be vertices that form a triangle in $P_{\ell n}^{k}$. Since there is no blue $(6,3,6)$-connector between $H^{\prime}(u), H^{\prime}(v)$, and $H^{\prime}(w)$, there are at most six $(2,1,2)$-connectors that do not overlap, two for each possibility to place the single vertex. These can be deleted by removing at most 10 vertices from each cluster.

By the above argument, we have to delete at most $4(2 k)+10\left(4 k^{2}\right) \leq t-5$ vertices from every cluster to get rid of all $(2,2)$-connectors and $(2,1,2)$-connectors. Let $H^{*}\left(v_{i}\right) \subseteq H^{\prime}\left(v_{i}\right)$ be the remainder of the corresponding cluster in $H^{\prime}$, and note that $\left|H^{*}\left(v_{i}\right)\right| \geq 5$ for $i=1, \ldots, \ell$.

A tuple $(u, v)$ is an end-tuple of a tight path with at least 4 vertices if $u$ and $v$ are consecutive vertices in the path and $u$ is contained in exactly two edges and $v$ is contained in exactly one. The two tuples $(u, v)$ and $(v, w)$ are the end-tuples of the tight path $(u, v, w)$ of length 3 . Furthermore, every tuple $(u, v)$ is an end-tuple of the tight path $(u, v)$ of length 2 .

Definition 8. For $i=1, \ldots, n-1$ we say that the quadruple $\left(u_{1}, u_{2}, w_{1}, w_{2}\right)$ satisfies property $Q_{i}$ if the following conditions hold:

1. $u_{1}, u_{2}, w_{1}, w_{2}$ are distinct vertices from $H^{\prime}$ such that the pairs $u_{1}, u_{2}$ and $w_{1}, w_{2}$ are in clusters $H_{r}, H_{s} \in\left\{H^{*}\left(v_{(i-1) \ell+1}\right), \ldots, H^{*}\left(v_{i \ell}\right)\right\}$, respectively, where $r \neq s$;

2. each of $\left(u_{1}, u_{2}\right)$ and $\left(w_{1}, w_{2}\right)$ is an end-tuple of a red tight path of length at least $i+1$ with vertices in $H^{*}\left(v_{1}\right) \cup \cdots \cup H^{*}\left(v_{i \ell}\right)$.

To prove Claim 7, i.e., construct $P_{n}^{(3)}$ in red, it is then sufficient to construct a quadruple satisfying property $Q_{n-1}$. We will construct this quadruple inductively. The base case $Q_{1}$ asks for two paths of length 2 and, therefore, it is enough to choose any pair $u_{1}, u_{2}$ from $H^{*}\left(v_{1}\right)$ and $w_{1}, w_{2}$ from $H^{*}\left(v_{2}\right)$. Therefore, the following is immediate:

There exists a quadruple $\left(u_{1}, u_{2}, w_{1}, w_{2}\right)$ for which $Q_{1}$ holds.

We will inductively find a quadruple $\left(u_{1}, u_{2}, w_{1}, w_{2}\right)$ satisfying property $Q_{i}$ for every $i=2, \ldots, n-1$. Ultimately, after $n$ steps, this gives us $P_{n}^{(3)}$ in red in $H^{\prime}$. Suppose $\left(u_{1}, u_{2}, w_{1}, w_{2}\right)$ satisfies property $Q_{i-1}$ for some $1<i \leq n-1$. In the inductive step, we obtain $\left(x_{1}, x_{2}, y_{1}, y_{2}\right)$ satisfying property $Q_{i}$ by extending one of the paths ending in $\left(u_{1}, u_{2}\right)$ or $\left(w_{1}, w_{2}\right)$ to get two longer paths ending in $\left(x_{1}, x_{2}\right)$ and $\left(y_{1}, y_{2}\right)$. This 


\section{J. Han, Y. Kohayakawa, S. Letzter, G. O. Mota and O. Parczy K}

mainly relies on the absence of $(2,2)$-connectors and $(2,1,2)$-connectors in blue and that there are $\ell$ clusters $H^{*}\left(v_{(i-1) \ell+1}\right), \ldots, H^{*}\left(v_{i \ell}\right)$ to choose $x_{1}, x_{2}, y_{1}, y_{2}$ from. As $k \geq 2 \ell$, all edges are present between these clusters and the clusters $H_{r}$ and $H_{s}$ containing $u_{1}, u_{2}$ and $w_{1}, w_{2}$, respectively.

Fact 9. Let $1<i \leq n-1$ and suppose $\left(u_{1}, u_{2}, w_{1}, w_{2}\right)$ satisfies property $Q_{i-1}$. Then there is a quadruple $\left(x_{1}, x_{2}, y_{1}, y_{2}\right)$ that satisfies property $Q_{i}$.

Proof. We will find $\left(x_{1}, x_{2}, y_{1}, y_{2}\right)$ following the strategy sketched above. Since there are no blue $(2,2)$ connectors between the clusters corresponding to $u_{1}, u_{2}$ and $w_{1}, w_{2}$, and all possible triples between these clusters are edges in $H^{\prime}$, then either the triple $u_{1} u_{2} w_{2}$ or the triple $w_{1} w_{2} u_{2}$ is red, say, w.l.o.g., $u_{1} u_{2} w_{2}$ is red. We let the red path of length at least $i$ that ends in $\left(u_{1}, u_{2}\right)$ be called $P_{\text {red }}$ and note that the triple $u_{1} u_{2} w_{2}$ already extends this path. We will show that it is possible to further extend this path to obtain two longer red tight paths with ends $\left(x_{1}, x_{2}\right)$ and $\left(y_{1}, y_{2}\right)$, respectively, such that $\left(x_{1}, x_{2}, y_{1}, y_{2}\right)$ satisfies property $Q_{i}$.

Notice that, as $\ell \geq 17$, by the pigeonhole principle there are nine sets $X_{1}, \ldots, X_{9}$ each contained in a different cluster from $H^{*}\left(v_{(i-1) \ell+1}\right), \ldots, H^{*}\left(v_{i \ell}\right)$ and of size $\left|X_{j}\right| \geq 3$ for $j \in[9]$ (here we use that $\left.\left|H^{*}\left(v_{i}\right)\right| \geq 5\right)$ such that either

$$
\text { for all } j \in[9] \text { and every } x \in X_{j} \text { the triple } u_{2} w_{2} x \text { is red }
$$

or

$$
\text { for all } j \in[9] \text { and every } x \in X_{j} \text { the triple } u_{2} w_{2} x \text { is blue. }
$$

We first consider the case where (4.2) holds. It is enough to assume that we have $X_{1}, \ldots, X_{5}$ and $\left|X_{j}\right| \geq 2$ for $j \in[5]$. If there are two sets $X, Y$ from $X_{1}, \ldots, X_{5}$ and $x_{1}, x_{2} \in X, y_{1}, y_{2} \in Y$ such that the triples $w_{2} x_{1} x_{2}$ and $w_{2} y_{1} y_{2}$ are red, then the quadruple $\left(x_{1}, x_{2}, y_{1}, y_{2}\right)$ satisfies property $Q_{i}$ as we can obtain two longer red paths by extending $P_{\text {red }}$ following $u_{1} u_{2} w_{2} x_{1} x_{2}$ and $u_{1} u_{2} w_{2} y_{1} y_{2}$, respectively. Otherwise, there are two sets $X, Y$ and $x_{1}, x_{2} \in X, y_{1}, y_{2} \in Y$ such that the triples $w_{2} x_{1} x_{2}$ and $w_{2} y_{1} y_{2}$ are blue. As there is no blue $(2,1,2)$-connector, the triple $w_{2} x_{1} y_{1}$ is red. There is no blue $(2,2)$-connector between the corresponding clusters, so either the triple $x_{1} y_{1} y_{2}$ or $y_{1} x_{1} x_{2}$ is red, say w.l.o.g. $x_{1} y_{1} y_{2}$ is red. This extends $P_{\text {red }}$ by following $u_{1} u_{2} w_{2} x_{1} y_{1} y_{2}$ and gives the end-tuple $\left(y_{1}, y_{2}\right)$. Repeating the same argument, which is possible, because there were five sets available (sets $\left.X_{1}, \ldots, X_{5}\right)$, we get an end-tuple $\left(z_{1}, z_{2}\right)$ that extends $P_{\text {red }}$ to a longer red path and, thus, a quadruple $\left(y_{1}, y_{2}, z_{1}, z_{2}\right)$ satisfying $Q_{i}$.

In the case where (4.3) holds we proceed as follows. As for all $j \in[9]$ there is no blue $(2,1,2)$ connector between the clusters of $u_{1}, u_{2}$ and $w_{1}, w_{2}$ and $X_{j}$, we have for every $x \in X_{j}$ that either the triple $w_{1} w_{2} x$ or the triple $u_{1} u_{2} x$ is red. Then we can assume by the pigeonhole principle w.l.o.g. (we will not use the triple $\left.u_{1} u_{2} w_{1}\right)$ that there are sets $X_{j}^{\prime} \subseteq X_{j}$ with $\left|X_{j}^{\prime}\right| \geq 2$ for $j \in[5]$ (here we use that $\left|X_{j}\right| \geq 3$ ) such that

$$
\text { for all } j \in[5] \text { and every } x \in X_{j}^{\prime} \text { the triple } w_{1} w_{2} x \text { is red. }
$$

Now we can continue exactly as in the case where (4.2) holds, with $u_{1} u_{2}$ replaced by $w_{1} w_{2}$ throughout and extending the path with end-tuple $\left(w_{1}, w_{2}\right)$. Observing that the red tight paths that we have constructed have length at least $i+1$, we see that Fact 9 is proved.

Fact 9 together with (4.1) finishes the proof of Claim 7 and hence the proof of Theorem 1 is complete. 
The Size-RAmsey Number of 3-Uniform Tight PATHS

\section{References}

[1] N. Alon and F. R. K. Chung, Explicit construction of linear sized tolerant networks, Discrete Math. 72 (1988), no. 1-3, 15-19. 2

[2] D. Bal and L. DeBiasio, New lower bounds on the size-Ramsey number of a path, 2019, arXiv:1909.06354. 2

[3] József Beck, On size Ramsey number of paths, trees, and circuits. I, J. Graph Theory 7 (1983), no. 1, $115-129.2$

[4] Ido Ben-Eliezer, Michael Krivelevich, and Benny Sudakov, The size Ramsey number of a directed path, J. Combin. Theory Ser. B 102 (2012), no. 3, 743-755. 4

[5] S. Berger, Y. Kohayakawa, G. S. Maesaka, T. Martins, W. Mendonça, G. O. Mota, and O. Parczyk, The size-Ramsey number of powers of bounded degree trees, J. Lond. Math. Soc. (2020), 1-19. 2

[6] Béla Bollobás, Extremal graph theory with emphasis on probabilistic methods, CBMS Regional Conference Series in Mathematics, vol. 62, Published for the Conference Board of the Mathematical Sciences, Washington, DC; by the American Mathematical Society, Providence, RI, 1986. 2

[7] D. Clemens, M. Jenssen, Y. Kohayakawa, N. Morrison, G. O. Mota, D. Reding, and B. Roberts, The size-Ramsey number of powers of paths, J. Graph Theory 91 (2019), no. 3, 290-299. 2, 3

[8] Andrzej Dudek, Steven La Fleur, Dhruv Mubayi, and Vojtech Rödl, On the size-ramsey number of hypergraphs, Journal of Graph Theory 86 (2017), no. 1, 104-121. 2

[9] Andrzej Dudek and Paweł Prałat, An alternative proof of the linearity of the size-Ramsey number of paths, Combin. Probab. Comput. 24 (2015), no. 3, 551-555. 2

[10] _ On some multicolor Ramsey properties of random graphs, SIAM J. Discrete Math. 31 (2017), no. 3, 2079-2092. 2

[11] P. Erdôs, On the combinatorial problems which I would most like to see solved, Combinatorica 1 (1981), no. 1, 25-42. 2

[12] J. Han, M. Jenssen, Y. Kohayakawa, G. O. Mota, and B. Roberts, The multicolour size-Ramsey number of powers of paths, J. Combin. Theory Ser. B 145 (2020), 359-375. 2

[13] M. Krivelevich, Long cycles in locally expanding graphs, with applications, Combinatorica (2018). To appear. 2

[14] Shoham Letzter, Path Ramsey number for random graphs, Combin. Probab. Comput. 25 (2016), no. $4,612-622.2$

[15] Linyuan Lu and Zhiyu Wang, On the size-ramsey number of tight paths, SIAM Journal on Discrete Mathematics 32 (2018), no. 3, 2172-2179. 2 


\section{AUTHORS}

Jie Han

School of Mathematics and Statistics

Beijing Institute of Technology

Beijing, China

jie_han@uri.edu

Yoshiharu Kohayakawa

Instituto de Matemática e Estatística

Universidade de São Paulo

Rua do Matão 1010, 05508-090 São Paulo, Brazil

yoshi@ime.usp.br

Shoham Letzter

Department of Mathematics

University College London

Gower Street, London WC1E 6BT

s.letzter@ucl.ac.uk

Guilherme Oliveira Mota

Instituto de Matemática e Estatística

Universidade de São Paulo

Rua do Matão 1010, 05508-090 São Paulo, Brazil

mota@ime.usp.br

Olaf Parczyk

London School of Economics

Department of Mathematics

Houghton Street, London, WC2A 2AE, UK

o.parczyk@1se.ac.uk 